\title{
A case of (double) ANCA-negative granulomatosis with polyangiitis (Wegener's)
}

\author{
Tetsuya Kashiwagi $\cdot$ Naoaki Hayama $\cdot$ Emiko Fujita $\cdot$ \\ Keiko Hara • Akiko Mii • Yukinari Masuda • \\ Yasuhiko Iino $\cdot$ Akira Shimizu $\cdot$ Yasuo Katayama
}

Received: 26 October 2011 / Accepted: 22 May 2012/ Published online: 26 June 2012

(C) Japanese Society of Nephrology 2012

\begin{abstract}
A 60-year-old man had experienced cough, bloody sputum, and a $38{ }^{\circ} \mathrm{C}$ fever for 1.5 months. He visited an outpatient clinic and received antibiotics and nonsteroidal anti-inflammatory drugs. However, because the symptoms continued, he visited our hospital. The past medical history included chronic sinusitis, hypertension, and diabetes mellitus. A chest x-ray film and computed tomography showed multiple pulmonary nodules with cavities. Macrohematuria had developed 3 days before admission, and renal function had deteriorated (creatinine, $2.45 \mathrm{mg} / \mathrm{dL}$ ) in 2 weeks. He was admitted to our hospital because of rapidly progressive glomerulonephritis (RPGN) and multiple pulmonary nodules. On admission, the clinical diagnosis was suspected to be granulomatosis with polyangiitis (Wegener's) (GPA), although tests for proteinase-3 anti-neutrophil cytoplasmic antibody (PR3-ANCA) were negative. Antibiotics were administered for 5 days. After renal biopsy, methylprednisolone pulse therapy and cyclophosphamide pulse therapy were performed. The pathological diagnosis on the basis of the renal biopsy was glomerular and interstitial hemorrhage, possibly associated with vasculitis. After the treatment, the pulmonary symptoms, multiple pulmonary nodules, and severe inflammatory reactions in the peripheral blood were resolved. However, renal dysfunction progressed to endstage renal disease 1 month after renal biopsy. Hemodialysis
\end{abstract}

T. Kashiwagi $(\square) \cdot$ N. Hayama $\cdot$ E. Fujita $\cdot$ K. Hara

A. Mii · Y. Iino - Y. Katayama

Department of Internal Medicine, Divisions of Neurology,

Nephrology, and Rheumatology, Nippon Medical School,

1-1-5 Sendagi, Bunkyo-ku, Tokyo 113-8603, Japan

e-mail: kashiwagi76@yahoo.co.jp; tk@nms.ac.jp

Y. Masuda · A. Shimizu

Department of Pathology, Analytic Human Pathology,

Nippon Medical School, Tokyo, Japan was started, and the steroid therapy was continued. During hemodialysis, a second renal biopsy was performed and led to a diagnosis of pauci-immune focal segmental crescentic glomerulonephritis. Renal function gradually recovered, and hemodialysis was discontinued. This case was (double) ANCA-negative GPA which presented prominent glomerular and interstitial hemorrhage, may be associated with small vessel vasculitis, but without active necrotizing and crescentic glomerular lesions, in the rapidly progressive glomerulonephritis.

Keywords Granulomatosis with polyangiitis (Wegener's) · Proteinase-3 anti-neutrophil cytoplasmic antibody $\cdot$ Rapidly progressive glomerulonephritis

\section{Introduction}

Granulomatosis with polyangiitis (Wegener's) (GPA) is a complex, immune-mediated disorder in which tissue injury results from the interplay of an initiating inflammatory event and a highly specific immune response. A part of this response is directed against previously shielded epitopes of neutrophil granule proteins, leading to high titers of autoantibodies known as anti-neutrophil cytoplasmic antibodies (ANCAs). Approximately 82-94\% of patients with either GPA or microscopic polyangiitis (MPA) are ANCA positive, depending on severity of the diseases $[1,2]$. GPA is primarily associated with proteinase 3 (PR3)-ANCA, while MPA is primarily associated with myeloperoxidase (MPO)-ANCA. However, $20 \%$ of patients with GPA or MPA have the alternative ANCA, and at least $10 \%$ of patients are ANCA negative [3-5]. ANCAs play an important role in the pathogenesis of GPA and related vasculitides. We have previously treated patients with 
ANCA-negative pauci-immune crescentic glomerulonephritis. Here we report a rare case of (double) ANCAnegative GPA we treated.

\section{Case report}

A 60-year-old man was admitted to our hospital with a 1.5-month history of cough, hemoptysis, and pyrexia. Although he had had hypertension and diabetes mellitus (DM) for 10 years, they had not been treated. The patient's mother had also had hypertension and DM. He visited a nearby outpatient clinic and received antibiotics and nonsteroidal anti-inflammatory drugs. However, the symptoms continued, and he visited our hospital 2 weeks later.

At that time, laboratory studies showed blood urea nitrogen (BUN), $19.8 \mathrm{mg} / \mathrm{dL}$; serum creatinine, $0.9 \mathrm{mg} / \mathrm{dL}$, and hemoglobin A1c (HbA1C), $9.4 \%$. Computed tomography (CT) of the chest revealed multiple pulmonary nodules with cavities (Fig. 1). In addition, macrohematuria had occurred 3 days before admission, and renal function had deteriorated to serum creatinine, $2.45 \mathrm{mg} / \mathrm{dL}$, in 2 weeks. The patient was then admitted to our hospital. Physical examination showed hypertension, coarse crackles in the left upper lobe, pitting edema of the lower limbs, and no sinusitis in the nasal cavities. Upper respiratory lesion was denied by CT and an otolaryngologist. Laboratory studies revealed leukocytosis with a high level of C-reactive protein, hyperglycemia, and elevated serum levels of creatinine and BUN. Serum levels of IgA and IgE were also high. Antinuclear antibodies (ANAs) and other antibodies, including antibodies against glomerular basement membrane, were not detected. In addition, PR3-ANCAs and MPO-ANCAs were not found. An indirect immunofluorescence test for ANCAs was also negative. Urinalysis showed $3+$ glucose, $1+$ protein $(670 \mathrm{mg} / \mathrm{dL})$, and \pm blood. The urine beta- 2 microglobulin level was elevated. Tests for tuberculosis and cultures of blood and urine were negative (Table 1). A sputum culture yielded bacteria of the resident flora. Bronchoscopic bronchoalveolar lavage yielded many neutrophils and alveolar macrophages. Transbronchial lung biopsy showed interstitial pneumonia with mild vasculitis and organization. However, granulomatous inflammatory lesions were not detected.

Antibiotics (ampicillin, $4 \mathrm{~g} / \mathrm{day}$, and clindamycin, $1.8 \mathrm{~g} /$ day) were administered for 5 days. After a renal biopsy, methylprednisolone pulse therapy ( $500 \mathrm{mg} /$ day for 3 days) and cyclophosphamide pulse therapy (700 $\mathrm{mg}$ for 1 day)

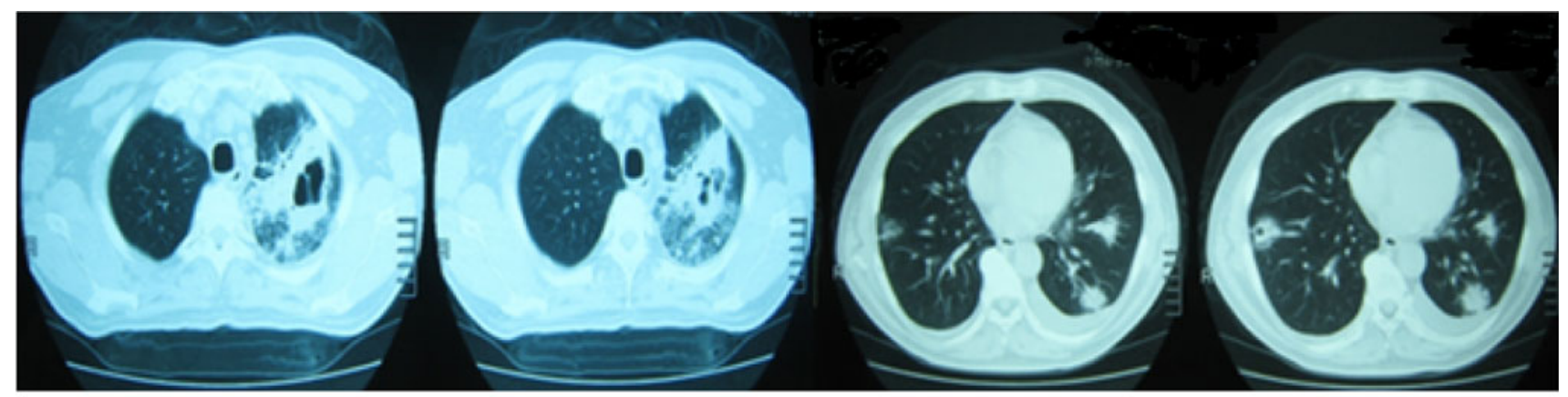

6 days before admission

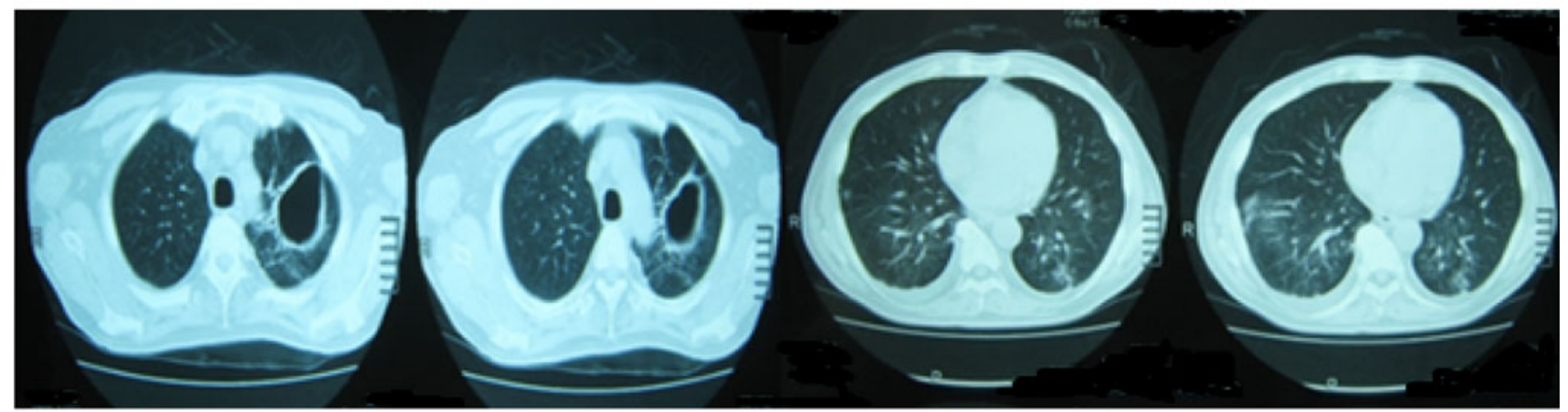

14 days after starting treatment

Fig. 1 Upper panel a CT scan obtained 6 days before admission shows consolidation with a cavity in the left upper lobe and multiple areas of consolidation without cavities in both lower lobes. Lower panel a CT scan obtained 14 days after the start of treatment shows that these lesions, other than the cavities, had improved with administration of prednisolone 
Table 1 The patient's laboratory data on admission

\begin{tabular}{|c|c|c|c|}
\hline \multicolumn{2}{|l|}{ Complete blood count } & \multicolumn{2}{|l|}{ Immunology } \\
\hline White blood cell & $18700 / \mu \mathrm{L}$ & Immunoglobulin $\mathrm{G}$ & $1691 \mathrm{mg} / \mathrm{dL}$ \\
\hline Neut Stab & $1.0 \%$ & Immunoglobulin A & $641 \mathrm{mg} / \mathrm{dL}$ \\
\hline Segmented & $88 \%$ & Immunoglobulin $\mathrm{M}$ & $49 \mathrm{mg} / \mathrm{dL}$ \\
\hline Lymphocyte & $4.0 \%$ & Immunoglobulin $\mathrm{E}^{\mathrm{a}}$ & $1010 \mathrm{IU} / \mathrm{mL}$ \\
\hline Eosinophil & $3.0 \%$ & Complement titer (CH50) & $50 \mathrm{U} / \mathrm{mL}$ \\
\hline Red blood cell & $509 \times 10^{4} / \mu \mathrm{L}$ & Complement C3 & $156 \mathrm{mg} / \mathrm{dL}$ \\
\hline Hemoglobin & $15.5 \mathrm{~g} / \mathrm{dL}$ & Complement $\mathrm{C} 4$ & $33 \mathrm{mg} / \mathrm{dL}$ \\
\hline Hematocrit & $44.5 \%$ & Immune complex $\mathrm{C} 1 \mathrm{q}$ & $<1.5$ \\
\hline Platelets & $23.6 \times 10^{4} / \mu \mathrm{L}$ & RAPA & $<40$ \\
\hline \multicolumn{2}{|l|}{ Blood chemical } & Antinuclear antibody & $<40$ \\
\hline Aspartate aminotransferase & 39 IU/L & Anti-ds-DNA antibody & Negative \\
\hline Alanine aminotransferase & $49 \mathrm{IU} / \mathrm{L}$ & Anti-SS-A antibody & Negative \\
\hline Lactate dehydrogenase & $271 \mathrm{IU} / \mathrm{L}$ & Anti-SS-B antibody & Negative \\
\hline$\gamma$-GTP & $64 \mathrm{U} / \mathrm{L}$ & Anti-GBM antibody & Negative \\
\hline Creatine phosphokinase & $56 \mathrm{U} / \mathrm{L}$ & Cryoglobulin & Negative \\
\hline Total cholesterol & $164 \mathrm{mg} / \mathrm{dL}$ & \multicolumn{2}{|l|}{$<$ ELISA $>$} \\
\hline Triglyceride & $120 \mathrm{mg} / \mathrm{dL}$ & PR3-ANCA & $<10$ \\
\hline Sodium & $132 \mathrm{mEq} / \mathrm{L}$ & MPO-ANCA & $<10$ \\
\hline Chloride & $97 \mathrm{mEq} / \mathrm{L}$ & \multicolumn{2}{|l|}{$<$ Indirect immunofluorescence $>$} \\
\hline Potassium & $5.0 \mathrm{mEq} / \mathrm{L}$ & ANCA & Negative \\
\hline Uric acid & $3.3 \mathrm{mg} / \mathrm{dL}$ & \multicolumn{2}{|l|}{ Culture tests } \\
\hline Blood urea nitrogen & $33.3 \mathrm{mg} / \mathrm{dL}$ & \multicolumn{2}{|c|}{$<$ Tuberculosis test (sputum and gastric sample) $>$} \\
\hline Creatinine & $2.45 \mathrm{mg} / \mathrm{dL}$ & Smear & Negative \\
\hline Total protein & $8.3 \mathrm{~g} / \mathrm{dL}$ & Polymerase chain reaction & Negative \\
\hline Albumin & $3.6 \mathrm{~g} / \mathrm{dL}$ & MAC & Negative \\
\hline C-reactive protein & $17.67 \mathrm{mg} / \mathrm{dL}$ & \multicolumn{2}{|l|}{$<$ Blood and urine culture $>$} \\
\hline Glucose & $217 \mathrm{mg} / \mathrm{dL}$ & Stenotrophomonas maltophilia & $3+$ \\
\hline Hemoglobin A1c & $9.4 \%$ & Acinetobacter baumannii & $2+$ \\
\hline \multicolumn{2}{|l|}{ Urinalysis } & \multicolumn{2}{|l|}{ Bronchoscopy ${ }^{\mathrm{b}}$} \\
\hline $\mathrm{pH}$ & 6.0 & Bronchial Lavage & Class IIIa $^{\mathrm{c}}$ \\
\hline Glucose & $3+$ & Neutrophil & Many \\
\hline \multirow[t]{2}{*}{ Protein } & $1+$ & Alveolar macrophage & $3+$ \\
\hline & $670 \mathrm{mg} / \mathrm{dL}, 900 \mathrm{mg} / \mathrm{day}$ & Transbronchial lung biopsy & Interstitial pneumonia $^{\mathrm{d}}$ \\
\hline Occult blood & \pm & & \\
\hline Erythrocytes & $1-4 / \mathrm{HPF}$ & & \\
\hline Leukocytes & $5-9 / \mathrm{HPF}$ & & \\
\hline Epithelial & $<1 / \mathrm{HPF}$ & & \\
\hline Cast & Negative & & \\
\hline NAG activity & $9.3 \mathrm{U} / \mathrm{L}$ & & \\
\hline Beta2-microglobulin & $3515 \mu \mathrm{g} / \mathrm{L}$ & & \\
\hline Cytodiagnosis & No heteromorphous cell & & \\
\hline
\end{tabular}

$\gamma$-GTP gamma-glutamyl transpeptidase, NAG N-acetyl-beta-D-glucosaminidase, RAPA rheumatoid arthritis particle agglutination, ds-DNA double stranded deoxyribonucleic acid, GBM glomerular basement membrane, PR3-ANCA proteinase-3 anti-neutrophil cytoplasmic antibody, MPO-ANCA myeloperoxidase anti-neutrophil cytoplasmic antibody, ANCA anti-neutrophil cytoplasmic antibody, MAC Mycobacterium aviumintracellulare complex

${ }^{a}$ Determined by the radioimmunosorbent test (RIST) method

b On day 7

c With inflammatory change

${ }^{d}$ With mild vasculitis and organization (no necrotic change and granuloma) 
were administered. The pathological diagnosis based on the renal biopsy was glomerular and interstitial hemorrhage, possibly associated with vasculitis, no necrosis or crescent formation in the glomeruli, and no granulomatous lesions (Fig. 2). This patient had DM, and several arteries showed severe hyaline arteriolosclerosis and polar vasculosis, but there was no evidence of arteritis (Fig. 3).

The symptoms (cough and hemoptysis), multiple pulmonary nodules visualized on $\mathrm{CT}$, and severe inflammatory reactions in the peripheral blood resolved after treatment; however, renal dysfunction progressed to end-stage renal disease 1 month after renal biopsy. Hemodialysis was started, and steroid therapy was continued $(60 \mathrm{mg} /$ day for 4 weeks, and then tapered by $5-10 \mathrm{mg} /$ week). During hemodialysis, a second renal biopsy was performed.

We diagnosed pauci-immune focal segmental crescentic glomerulonephritis (Fig. 4). Renal function had gradually recovered, and hemodialysis was discontinued. The most recent measures of renal function showed BUN, 40.5 $\mathrm{mg} / \mathrm{dL}$, and creatinine, $2.61 \mathrm{mg} / \mathrm{dL}$, without hemodialysis.
A summary of the clinical course of the present case is shown on Fig. 5.

\section{Discussion}

GPA is a systemic granulomatous angiitis whose main symptoms are crescentic glomerulonephritis and necrotizing granulomatous vasculitis of the lung and upper respiratory tract. In its early stage, as upper respiratory tract impairment progresses, purulent nasal discharge and nasal bleeding develop and lead to nasal cavity ulceration and nasal septum perforation. With further progression, the characteristic symptom of GPA—saddle-nose deformityappears. In addition to these symptoms, purulent otitis media, episcleritis, and exophthalmos can develop. Lung lesions are found in $85 \%$ of patients and cause cough, hemoptysis, and respiratory distress. Renal lesions develop in $80 \%$ of patients within 2 years of onset. Moreover, purpura of the lower limbs and mononeuritis multiplex are
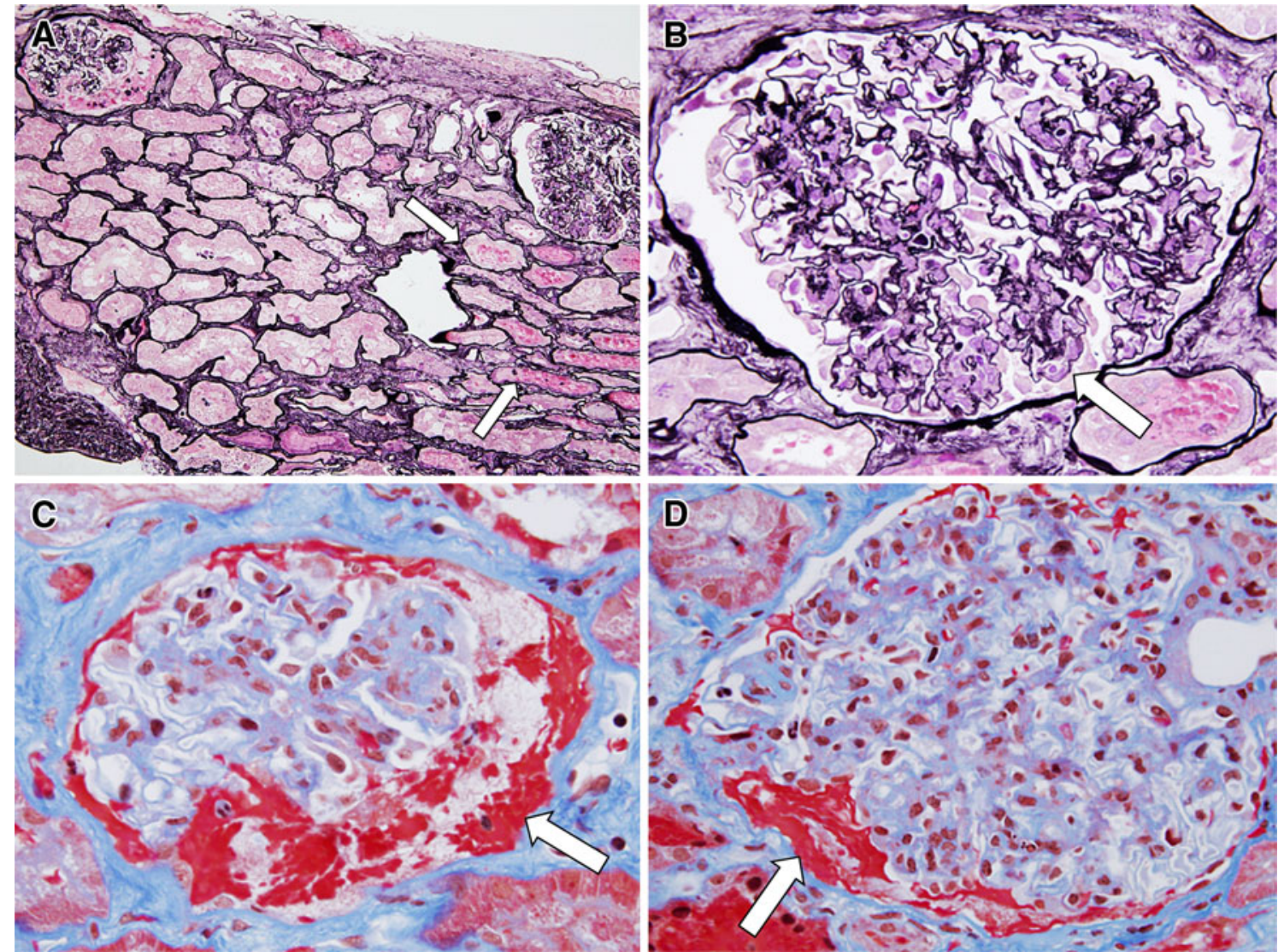

Fig. 2 Light microscopic findings of glomeruli from 1st biopsy samples (a, b PAM stain; c, d Masson stain; a $\times 200$; b-d: $\times 600)$. The biopsy specimens contained 9 glomeruli in which 2 glomeruli were obsolescent. Massive red blood cell casts were present in the renal tubules (arrow in a). In 7 glomeruli, 5 glomeruli showed mild segmental endocapillary proliferation (arrow in b) with infiltration of

neutrophils and monocytes. 3 glomeruli showed hemorrhage (arrow in $\mathbf{c}$ and d) in Bowman's space. No necrotizing or crescentic lesions were detected in glomeruli. Immunoflourescence study showed no obvious deposition of immunoglobulin (Ig) G, IgA, IgM, and complement (C)3, C1q, and $\mathrm{C} 4$ in glomeruli, indicating pauciimmune type of deposition 

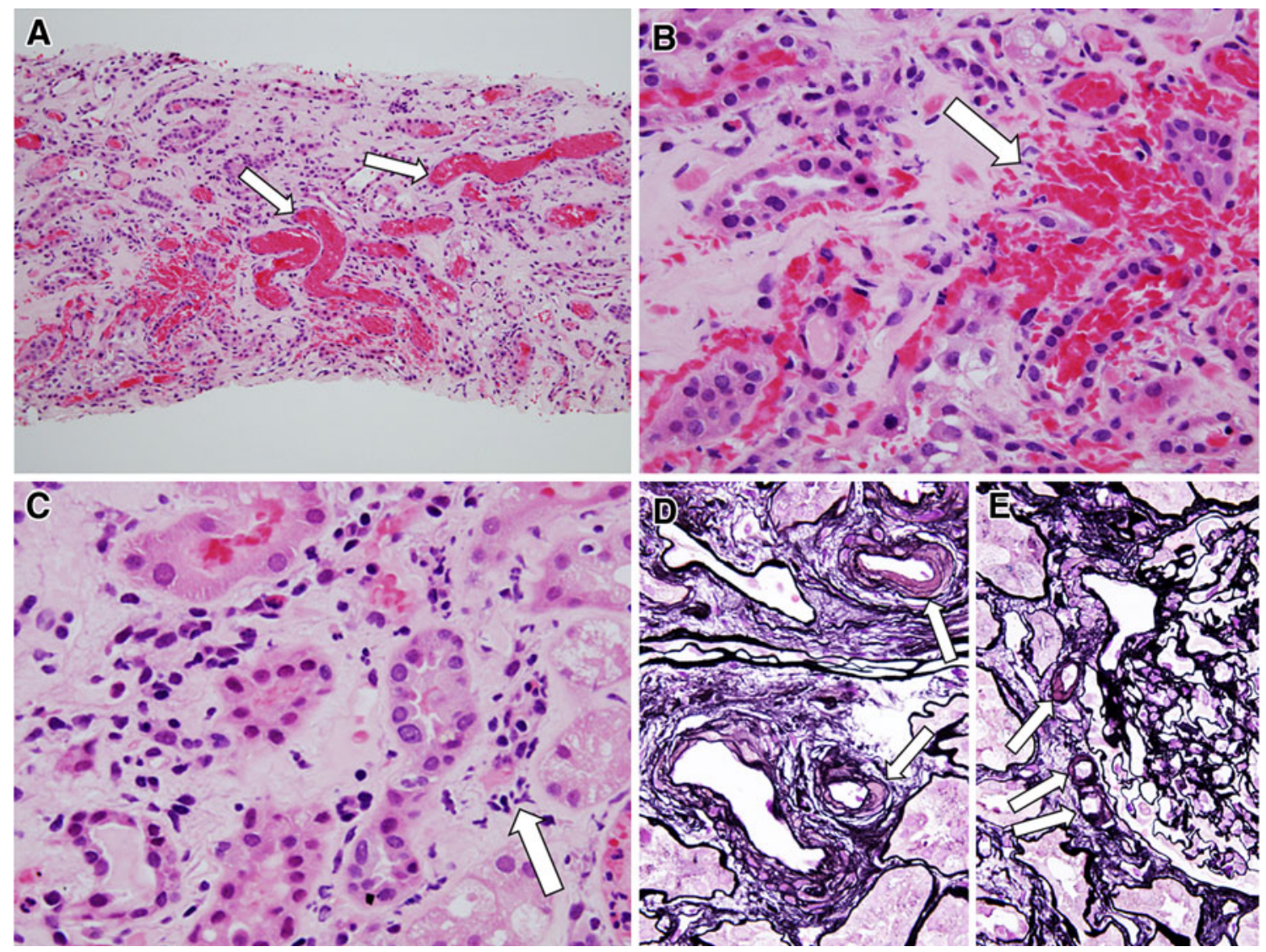

Fig. 3 Light microscopic findings of tubulointerstitium and arterioles from 1st biopsy samples (a-c H\&E stain; d, e: PAM stain; a $\times 200$; $\mathbf{b}, \mathbf{c}, \mathbf{e} \times 600 ; \mathbf{d} \times 400)$. In interstitium, many red blood cell casts were present in tubular lumens (arrow in a), may be associated with glomerular hemorrhage. Localized interstitial hemorrhage was also noted with peritubular capillaritis (arrow in b). Peritubular capillaritis

found in association with vasculitis. The diagnostic criteria of the Ministry of Health, Labour and Welfare (MHLW) of Japan, the classification criteria of the American College of Rheumatology (ACR) and the European Medicines Agency (EMA) algorithm are often used to diagnose GPA. These definitions have gained wide acceptance in defining vasculitides by pathology, including the size of affected vessels and the organ systems involved.

In the present case, the patient had rapidly progressive renal failure and multiple pulmonary nodules, which are found in GPA, but PR3-ANCAs were absent. A second biopsy showed pauci-immune crescentic glomerulonephritis. Therefore, this case falls into the "definite" category of the diagnostic criteria for GPA of the MHLW of Japan. With the ACR classification criteria, this case would be diagnosed as GPA because of the findings of urinalysis and chest radiography. In this instance, the sensitivity was $88 \%$, and the specificity was $92.0 \%$ [6]. In the EMA algorithm, fixed infiltrates that are present on chest X-ray for over 1 month fall into a classification of GPA; thus, this case would be

was expanded in the renal cortex and medulla with or without interstitial hemorrhage (arrow in c). This patient had DM, and severe hyalinosis of small arterioles (arrow in d), and polar vasculosis around glomerular hilus (arrow in e) were evident although nodular lesions of DM nephropathy could not be detected in glomeruli. No necrotizing or granulomatous vasculitis was noted in arteries

diagnosed as GPA. The diagnostic criteria of the MHLW of Japan indicate that the PR3-ANCAs will be positive at high rates in its main examination findings. ANCA is an autoantibody mainly recognizing antigens on azurophilic granules in the neutrophil cytoplasm and is classified, from the staining pattern, as PR3-ANCA or MPO-ANCA. Approximately $82-94 \%$ of patients with either GPA or microscopic polyangiitis (MPA) are ANCA positive, depending on severity of disease [1,2]. GPA is primarily associated with PR3-ANCA, while MPA is primarily associated with MPOANCA. However, $20 \%$ of patients with GPA or MPA have the alternative ANCA, and at least $10 \%$ of patients are ANCA negative [3-5]. In many cases, the ANCA titer will decrease or be 0 with remission induction therapy $[4,6,7]$. The main pathologic difference between GPA and MPA is the absence of granulomatous inflammation in MPA. Routine laboratory tests are generally nonspecific in GPA $[8,9]$. The diagnosis of GPA is based on clinical and laboratory findings. There is clearly a small subset of patients with active, generalized GPA whose ANCAs are absent. 

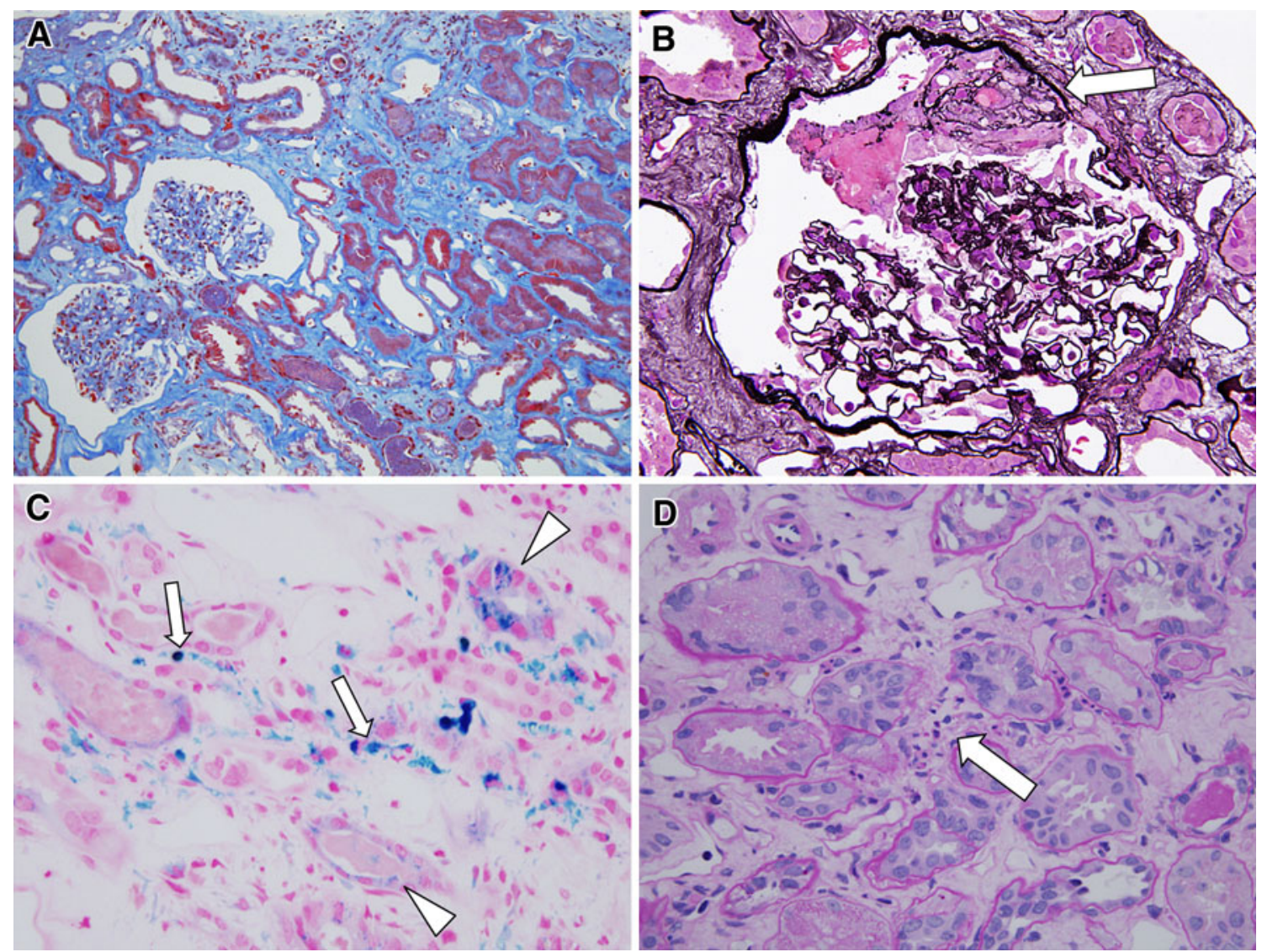

Fig. 4 Light microscopic findings from 2nd biopsy samples (a Masson stain; b PAM stain; c Berlin blue stain; d H\&E stain; a $\times 200$; b-d $\times 600$ ). The biopsy specimens contained 19 glomeruli in which 1 glomerulus showed obsolescence. Interstitial hemorrhage and red blood cell (RBC) casts were disappeared from the renal tubules and interstitium. In the glomeruli, endocapillary proliferative or necrotizing lesions could not be detected, but 3 glomeruli were accompanied

Many cases of ANCA-negative GPA are of the limited type [10-13]. Some cases of ANCA-negative GPA show pathological changes in the kidney, but these cases tend to turn ANCA-positive during the course [14]. On the other hand, in the present GPA case, ANCAs did not appear as the disease progressed. The reason for the absence of ANCAs has been not clear. Patients with GPA demonstrate abnormalities in the number and function of regulatory $\mathrm{T}$ cells (increased fraction of CD $4{ }^{+} \mathrm{CD} 25^{+}$T cells); $\mathrm{CD} 4{ }^{+} \mathrm{CD} 25^{\mathrm{hi}} \mathrm{T}$ cells were able to suppress $\mathrm{T}$-cell proliferation to PR3 in healthy controls and ANCA-negative patients but not in ANCA-positive patients [15]. A skewed response of an interleukin-17-producing T-helper cell lineage (Th17) found in patients with ANCA-positive GPA following stimulation with the autoantigen PR3 suggests that interleukin-17 is involved in disease pathogenesis [16].

Moreover, the response to the medical treatment of ANCA-negative GPA varies: some cases show a good response to prednisolone or cyclophosphamide, but other

by fibrocellular crescents (arrow in b), and 1 glomerulus had fibrous crescent. Berlin blue stain indicated the deposition of hemosiderin in renal tubular epithelial cells (arrowhead in c) and interstitium (arrow in c), indicating that post-status of massive RBC casts in renal tubules and interstitial hemorrhage, respectively. Capillaritis was present in peritubular capillaries (arrow in d), but the degree of capillaritis was milder than 1st biopsy

cases show a poor response to these agents but are controlled with rituximab $[10,11]$. The responses to methylprednisolone and cyclophosphamide in the present case were good. Kidney biopsy typically reveals segmental necrotizing glomerulonephritis that is usually pauci-immune on immunofluorescence or electron microscopy [17-19].

In the present case, we could not rule out the possibility that the renal lesions had been caused by infection or drugrelated acute tubulointerstitial nephritis; however, the lesions were unlikely to have been caused by infection because the symptoms decreased through remission-induction therapy with cyclophosphamide and steroid therapy. Furthermore, the development of renal lesions due to druginduced nephritis would make less sense because no invasion by eosinophils was found in renal tissue. Therefore, we decided, on the basis of the first renal biopsy, that the pathological diagnosis was vasculitis in glomeruli and peritubular capillaries with hemorrhage. We diagnosed this case as GPA. Moreover, the following biopsy findings possibly 
Fig. 5 Clinical course of the present case. During the course, macrohematuria was observed only once 3 days before admission, but oliguria was not observed. After 1st renal biopsy, methylprednisolone pulse therapy ( $500 \mathrm{mg} /$ day for 3 days) and cyclophosphamide pulse therapy (700 mg for 1 day) were administered, but renal dysfunction progressed. So hemodialysis was started, and steroid therapy was continued $(60 \mathrm{mg} /$ day for 4 weeks and then tapered by $5-10 \mathrm{mg} /$ week). Renal function gradually recovered, and hemodialysis was discontinued. The most recent measures of renal function showed serum creatinine $2.61 \mathrm{mg} / \mathrm{dL}$, without hemodialysis

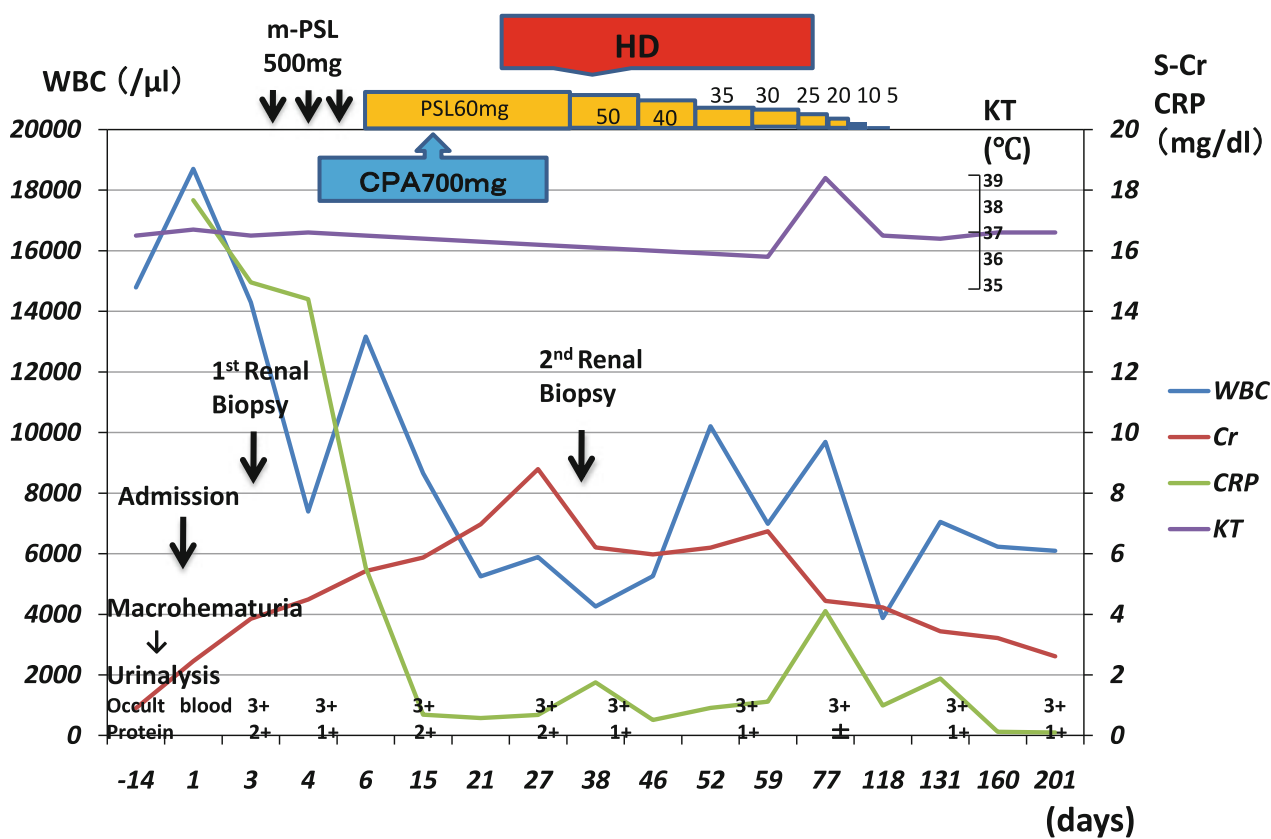

days after admission involves with the deterioration of renal functions. In the 1st biopsy sample, there found massive red blood cell (RBC) casts in the renal tubules and Bowman's space, which may be caused by glomerular hemorrhage. In addition, localized interstitial hemorrhage was also present that may be caused by peritubular capillaries. In the 2 nd biopsy sample, the deposition of hemosiderin in renal tubular epithelial cells and interstitium indicated that post-status of massive RBC casts in renal tubules and interstitial hemorrhage, respectively. Tubulointerstitial injury has been well known as the association with acute kidney injury (AKI). Furthermore, gross hematuria occurring in patients with underlying glomerular disease has been associated with the development of transient AKI. This association has been described in patients with IgA nephropathy and warfarin-related nephropathy thin basement membrane disease [20-24]. Taking account of these, glomerular hemorrhage, massive RBC casts, and peritubular capillaritis and interstitial hemorrhage may have involved with the onset of AKI in the present case.

This case was (double) ANCA-negative GPA which presented atypical course of RPGN caused by glomerular and interstitial hemorrhage without typical crescentic glomerulonephritis. Further careful observation of this case is necessary.

\section{References}

1. Guillevin L, Durand-Gasselin B, Cevallos R, Gayraud M, Lhote F, Callard P, et al. Microscopic polyangiitis: clinical and laboratory findings in eighty-five patients. Arthritis Rheum. 1999; 42(3):421-30.
2. Finkielman JD, Lee AS, Hummel AM, Viss MA, Jacob GL, Homburger HA, et al. ANCA are detectable in nearly all patients with active severe Wegener's granulomatosis. Am J Med. 2007; 120(7):643.e9-14.

3. Falk RJ, Jennette JC. ANCA small-vessel vasculitis. J Am Soc Nephrol. 1997;8(2):314-22.

4. Hagen EC, Daha MR, Hermans J, Andrassy K, Csernok E, Gaskin G, et al. Diagnostic value of standardized assays for antineutrophil cytoplasmic antibodies in idiopathic systemic vasculitis. EC/BCR Project for ANCA Assay Standardization. Kidney Int. 1998;53(3):743-53.

5. Savige J, Pollock W, Trevisin M. What do antineutrophil cytoplasmic antibodies (ANCA) tell us? Best Pract Res Clin Rheumatol. 2005;19(2):263-76.

6. Leavitt RY, Fauci AS, Bloch DA, Michel BA, Hunder GG, Arend WP, et al. The American College of Rheumatology 1990 criteria for the classification of Wegener's granulomatosis. Arthritis Rheum. 1990;33(8):1101-7.

7. Hoffman GS, Specks U. Antineutrophil cytoplasmic antibodies. Arthritis Rheum. 1998;41(9):1521-37.

8. Hoffman GS, Kerr GS, Leavitt RY, Hallahan CW, Lebovics RS, Travis WD, et al. Wegener's granulomatosis: an analysis of 158 patients. Ann Intern Med. 1992;116(6):488-98.

9. Seo P, Stone JH. The antineutrophil cytoplasmic antibody-associated vasculitides. Am J Med. 2004;117(1):39-50.

10. Khan A, Lawson CA, Quinn MA, Isdale AH, Green MJ. Successful treatment of ANCA-negative Wegener's granulomatosis with rituximab. Int J Rheumatol. 2010;. doi:10.1155/2010/846063.

11. Ooka S, Maeda A, Ito $H$, Omata $M$, Yamada $H$, Ozaki S. Treatment of refractory retrobulbar granuloma with rituximab in a patient with ANCA-negative Wegener's granulomatosis: a case report. Mod Rheumatol. 2009;19(1):80-3.

12. Greco P, Palmisano A, Vaglio A, Scoditti U, Antonelli MY, Crisi $\mathrm{G}$, et al. Meningeal involvement in apparently ANCA-negative Wegener's granulomatosis: a role for PR3 capture-ELISA? Rheumatology. 2007;46(8):1375-6.

13. Reinhold-Keller E, de Groot K, Holl-Ulrich K, Arlt AC, Heller $\mathrm{M}$, Feller AC, et al. Severe CNS manifestations as the clinical 
hallmark in generalized Wegener's granulomatosis consistently negative for antineutrophil cytoplasmic antibodies (ANCA). A report of 3 cases and a review of the literature. Clin Exp Rheumatol. 2001;19(5):541-9.

14. Aasarød K, Bostad L, Hammerstrøm J, Jørstad S, Iversen BM. Renal histopathology and clinical course in 94 patients with Wegener's granulomatosis. Nephrol Dial Transplant. 2001;16(5): 953-60.

15. Morgan MD, Day CJ, Piper KP, Khan N, Harper L, Moss PA, et al. Patients with Wegener's granulomatosis demonstrate a relative deficiency and functional impairment of T-regulatory cells. Immunology. 2010;130(1):64-73.

16. Abdulahad WH, Stegeman CA, Limburg PC, Kallenberg CG. Skewed distribution of Th17 lymphocytes in patients with Wegener's granulomatosis in remission. Arthritis Rheum. 2008; 58(7):2196-205.

17. Eisenberger U, Fakhouri F, Vanhille P, Beaufils H, Mahr A, Guillevin L, et al. ANCA-negative pauci-immune renal vasculitis: histology and outcomes. Nephrol Dial Transplant. 2005; 20(7):1392-9.

18. Hauer HA, Bajema IM, van Houwelingen HC, Ferrario F, Noël LH, Waldherr R, European Vasculitis Study Group (EUVAS), et al. Renal histology in ANCA-associated vasculitis: differences between diagnostic and serologic subgroups. Kidney Int. 2002; 61(1):80-9.

19. Haas M, Eustace JA. Immune complex deposits in ANCA-associated crescentic glomerulonephritis: a study of 126 cases. Kidney Int. 2004;65(6):2145-52.

20. Gutiérrez E, González E, Hernández E, Morales E, Martínez MA, Usera $\mathrm{G}$, et al. Factors that determine an incomplete recovery of renal function in macrohematuria-induced acute renal failure of IgA nephropathy. Clin J Am Soc Nephrol. 2007;2(1):51-7.

21. Kveder R, Lindic J, Ales A, Kovac D, Vizjak A, Ferluga D. Acute kidney injury in immunoglobulin A nephropathy: potential role of macroscopic hematuria and acute tubulointerstitial injury. Ther Apher Dial. 2009;13(4):273-7.

22. Brodsky SV, Satoskar A, Chen J, Nadasdy G, Eagen JW, Hamirani $\mathrm{M}$, et al. Acute kidney injury during warfarin therapy associated with obstructive tubular red blood cell casts: a report of 9 cases. Am J Kidney Dis. 2009;54(6):1121-6.

23. Kabir A, Nadasdy T, Nadasdy G, Hebert LA. An unusual cause of gross hematuria and transient ARF in an SLE patient with warfarin coagulopathy. Am J Kidney Dis. 2004;43(4):757-60.

24. Abt AB, Carroll LE, Mohler JH. Thin basement membrane disease and acute renal failure secondary to gross hematuria and tubular necrosis. Am J Kidney Dis. 2000;35(3):533-6. 